\title{
Tagungsbericht
}

\section{APE 2019: Wissenschaftliche Verlage auf der Suche nach Wertschöpfung}

\author{
„Platforms or Pipelines? Where is the Value in Scholarly Communications?“ lautete das \\ Motto der 14. Konferenz Academic Publishing in Europe (APE 2019) in Berlin vom
}

15. bis 16. Januar 2019

https://doi.org/10.1515/iwp-2019-2008

Bereits zum 14. Mal fand die Konferenz Academic Publishing in Europe in Berlin statt. Zur Tagung kamen rund 30 Referierende und 250 Teilnehmende aus dem wissenschaftlichen Verlagsbereich, Bibliotheken, Forschungseinrichtungen, Fachgesellschaften und Technologiefirmen, die zwei Tage teils lebhaft über aktuelle Trends und Veränderungen in der Wissenschaftskommunikation diskutierten. Seit Open Access steckt die Branche in einem starken Wandel, der in den letzten Jahren an Fahrt aufgenommen hat. Mehr und mehr Institutionen schließen sich der Idee an, öffentlich finanzierte Forschung auch öffentlich zugänglich zu machen.

Dieses Jahr wurden auf der APE in sieben thematischen Sessions über aktuelle wissenschaftspolitische und strategische Standpunkte zu Open Access und Open Science diskutiert, sowie über neue Wege aus der Krise des traditionellen Peer-Review-Verfahrens ${ }^{1}$. Technische Entwicklungen und Projekte von StartUps wurden unter dem Aspekt der besseren Vernetzung und Erschließung von Daten untersucht. Auch Entwicklungen beim E-Book standen auf der Agenda.

Spannend wurde es, als Judy Verses, Executive Vice President von Wiley, am ersten Konferenztag während ihrer Keynote dem Auditorium noch vor der offiziellen Pressekonferenz $z^{2}$ über den erfolgreichen Vertragsabschluss mit dem Projekt DEAL (einem Konsortium aus über 700 forschenden Einrichtungen in Deutschland) hinsichtlich

1 Vgl. https://www.forschung-und-lehre.de/suche-nach-gutachternwird-immer-schwieriger-1017/ [1.4.2019].

2 Vgl.https://www.projekt-deal.de/pressekonferenz-zum-deal-vertr ag-mit-wiley/ [1.4.2019].

*Kontaktperson: Elgin Helen Jakisch, U\&B Interim-Services, Berlin, E-Mail: jakisch@ub-interim.de einer neuen Open-Access-Strategie verkünden konnte. Die Symbiose zwischen Wissenschaft und Verlagswesen hat an diesem Tag eine neue Entwicklung genommen und möglicherweise weitreichende Folgen. Der Vertrag wurde auf der Konferenz von beiden Verhandlungspartnern bereits als revolutionär bezeichnet. Verses war überzeugt, dass Verlage den Wandel nur überleben, wenn sie ihn aktiv mitgestalteten und nicht von ihm überrollt würden. Die Geschäftsmodelle der Verlage müssten die Forschenden unterstützen. Dies ginge nicht ohne eine Langzeitstrategie und Partnerschaften. Offene Daten, offene Artikel und offene Standards sind nach Verses Ansicht für das Überleben der Branche essenziell.

Die anwesenden Vertreter der Verlage bemühten sich, ihre angestammte und neutrale Rolle bei der Wahrung der Integrität der Wissenschaftsdiskurse zu betonen. Man fokussierte sich auf den Vorteil der Unvoreingenommenheit, Forschungsdispute in ihrer Gesamtheit abzubilden und keinerlei Druck auf Herausgeber auszuüben. Dr. Michiel Kolman, Präsident der International Publishers Association, sieht Verlage in Zeiten von Fake News immer noch als Garanten von Informationsqualität und Hüter der Glaubwürdigkeit. Die Vertreter der Open-Access-Bewegung wiederum erneuerten ihre Argumente, dass öffentlich finanzierte Forschung öffentlich zugänglich sein müsse und davon ebenfalls die Glaubwürdigkeit der Wissenschaft abhinge. Man müsse, so einige Referenten, Open Access in einem größeren Zusammenhang sehen und dies in die Vision von Open Science strategisch einbetten.

\section{Full Open Access oder Diversität der Modelle?}

Die EU-Kommission unterstützt klar den Kurs der OpenAccess-Bewegung. Ihr Vertreter Robert-Jan Smits, Sonderberater beim Europäischen Zentrum für Politische Strate- 


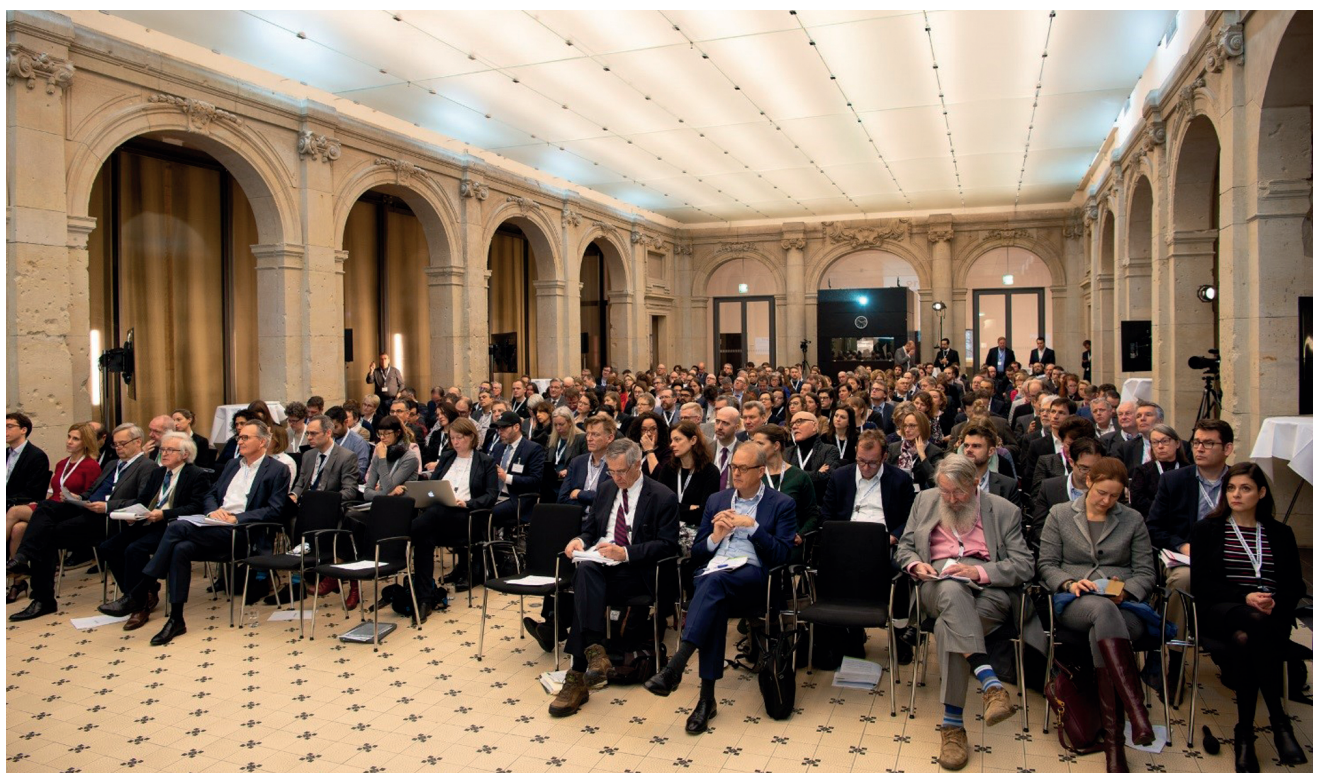

Abbildung 1: 250 Teilnehmende aus Wissenschaft, Verlagswesen, Bibliothek und Technologieanbietern diskutieren zwei Tage lang Trends im Verlagswesen. (Foto: Edwin de Kemp)

gie $^{3}$, einem Thinktank der EU-Kommission, forderte „full open access“ bis zum Jahr 2020 im Sinne der AO2020-Initiative 4 . Er erläuterte den „Plan S“, dem im September 2018 bereits elf forschungsfinanzierende Organisationen in Europa zugestimmt hatten ${ }^{5}$. Diese verpflichten sich zu Standards, wie u. a. das Publizieren in DOAJ (Directory of Open Access Journals) gelisteten OA-Zeitschriften, fairen Article-Processing-Charges (APCs) und Servicegebühren für das Peer-Review sowie die Verwendung der CC-BY 4.0 Creative-Commons-Lizenz und ethischen Grundsätzen des COPE (Commitee on Publication Ethics). Smits Standpunkt war deutlich. Künftig sei keine Bezahlschranke akzeptabel und die Verlage hätten sich aus Sicht der EU-Kommission bisher nicht genug bewegt.

Vertreter der STM-Verlage kritisierten in der anschließend kontroversen Diskussion, dass ihre Marktbedingungen von Plan S nicht respektiert würden. Wissenschaftler hätten nicht mehr die freie Wahl, wo sie veröffentlichen wollten. Wortmeldungen aus dem Publikum bemängelten die Ausrichtung von Plan S an der Veröffentlichungspraxis im naturwissenschaftlichen Bereich. Dies könne nicht als Blaupause für die Geisteswissenschaften und vor allem nicht für kleinere Verlage dienen, deren Existenz man

3 Vgl. https://ec.europa.eu/info/departments/european-political-str ategy-centre_de [1.4.2019].

4 Vgl. https://oa2020.org/ [1.4.2019].

5 Smits erwähnte, Deutschland sei nicht darunter, da es mit dem Projekt DEAL bereits eine Allianz der Wissenschaftsorganisationen gäbe, die im Sinne von Plan S und den Grundsätzen von OA2020 Linzenzverhandlungen mit Verlagen führe. nicht gefährden dürfe. Der EU-Sonderberater räumte ein, dass man mit einer Übergangszeit rechne, aber nicht vom bisherigen Kurs abweichen würde. Die Plan-S-Initiative wolle die hybriden Modelle bei der Transformation unterstützen. Auch hätten Nachwuchswissenschaftler am Plan $S$ mitgearbeitet.

Vertreter großer traditioneller Fachgesellschaften plädierten für eine Diversität verschiedener Modelle. Sie mahnten, dass das Publikationswesen nur eine Überlebenschance hätte, wenn alle Modelle unterstützt und keines ausgeschlossen würde. Sowohl die AAAS (American Association for the Advancement of Science), vertreten durch Dr. Rush Holt, Präsident und Herausgeber der Wissenschaftszeitschrift „Science“, als auch Dr. Bernd Pulverer (Head of Scientific Publication, EMBO Journal) und Dr. Robert Parker (CEO der Royal Society of Chemistry) griffen in ihren Statements nochmals die besondere Position der publizierenden Fachgesellschaften auf und gaben zu bedenken, dass OA-Zeitschriften laufend finanziert werden müssten, ebenso wie neueste digitale Standards von Artikeln oder KI-basierte Textanalysen.

\section{Mehr Daten, mehr Analysemöglichkeiten}

Bei einem anschließenden Panel zu Open Science referierte Prof. Dr. Gerard Meijer (Direktor des Fritz-Haber-Instituts Berlin und ebenfalls Verhandlungspartner beim Projekt DEAL) über die im Dezember 2017 stattgefundene 
14. Berliner Open Access Konferenz $z^{6}$, auf der die Teilnehmenden die Angleichung internationaler Strategien für Verhandlungen zwischen den Institutionen, Forschungsfonds und Verlagen diskutiert hatten. Er war überzeugt, dass wirtschaftliche Anpassungen vorgenommen würden, wenn sich der Markt Stück für Stück verändere. Da es letztlich bei Open Science um zukünftige Datenanalysen ginge, sei es wichtig, ergänzte Meijers, dass die forschenden Institutionen die Kontrolle über die Daten behalten und diese nicht an die Verlage abgeben.

Im Hinblick auf immer mehr Daten aus dem wissenschaftlichen Umfeld, wurden auf der Konferenz Lösungen diskutiert, die das gesamte Ökosystem der Forscher abbilden, wie am Beispiel der Plattform „Dimensions“, die als „Linked-Research-Data-Plattform“ verschiedene Publikationen und Informationen miteinander verbindet, um umfassende Überblicke über Themengebiete zu ermöglichen. Die EU fördert mit dem Programm Horizon $2020^{7}$ Projekte im Open-Science-Bereich, um Wissensverluste zu kompensieren. Die Open-Science-Bewegung sucht nach klaren Richtlinien und Standards für Formate, Rechte und Übertragungswege. Bisher gibt es für Forscher zu wenig Anreize für das Teilen von Daten, wie eine Studie von Figshare ${ }^{8}$ herausgefunden hat. Noch teilten viele nicht, weil sie ihr Wissen für sich behalten wollten. Eine Teilnehmerin aus dem $\mathrm{Pu}$ blikum wandte ein, dass je nachdem, wer das Teilen von Daten fordere, verschiedene Datengrundlagen entstehen könnten. Grundsätzlich geht die Bereitschaft zu Open Science mit einem Kulturwandel der Wissenschaftsdisziplinen einher.

\section{Lässt sich das Peer-Review-Verfahren reformieren?}

Der bisherige Peer-Review-Prozess scheint an seine Grenzen zu stoßen. Es herrscht ein Mangel an Gutachtern und ein ansteigendes Aufkommen an Publikationen. Ein Panel widmete sich diesem Thema genauer und diskutierte, ob unabhängige Vollzeitherausgeber statt der freiwilligen Peers eine bessere Qualität garantieren könnten, da sie keiner Einrichtung oder anderen Aufgabe verpflichtet seien. Ein offener Peer-Review-Prozess könnte auch Vorteile ha-

6 Vgl. hierzu https://oa2020.org/b14-conference/ [1.4.2019]. 7 Vgl. hierzu https://ec.europa.eu/programmes/horizon2020/ [1.4.2019].

8 Vgl. hierzu „The State of Open Data Report 2018“, https://figshare. com/articles/The_State_of_Open_Data_Report_2018/7195058 [1.4.2019]. ben, weil dieser der Community und dem Inhalt nütze. Offene Reviews könnten zitiert und in den Diskurs eingebunden werden. Bei Highwire tun sich 130 Nachwuchswissenschaftler als „Reviewer“ zu „Journal-Clubs“ zusammen und bewerten die Qualität der eingereichten Artikel offen. Letztlich bedürfe es ebenfalls eines Kulturwandels und Anreize für das Peer-Review sowie offene Diskussionen in den wissenschaftlichen Communities, so das Fazit des Panels.

\section{Gibt es zukünftig mehr Kooperationen?}

Die 14. APE setzte wie immer Schlaglichter auf hochaktuelle Trends im akademischen Verlagsbereich. Das Planungskomitee war von der Ausgangsfrage nach tragfähigen Plattformen, Services und Konzepten mitten in der Debatte um den richtigen Weg zur wertschöpfenden Erhaltung der wissenschaftlichen Informationsqualität bei gleichzeitiger Diversifizierung und Disruption des Marktes gelandet. Die Politik und Geldgeber der Forschung in den europäischen Ländern werden ebenso wie die Auswirkungen des Deals mit Wiley ausschlaggebend für weitere Veränderungen sein. Global verändert sich derzeit das Aufkommen an Literatur aus China, welches bereits das der USA überholt hat. Dieser Markt könnte eine ernstzunehmende Einflussgröße werden.

Künftige Modelle und Standards sind noch nicht abschließend ausgehandelt. Trotz aller kontroversen Debatten könnte der Wiley-Deal einen Trend zu mehr Zusammenarbeit auslösen. Die Verlage müssen mutiger werden, gewohnte Pfade zu verlassen. Die Digitalisierung verändert sowohl die Wissenschaften, als auch die Verlage und die Arbeit in den Bibliotheken erheblich. Die Stärke der Konferenz ist, die Entwicklungen dieser Auswirkungen fortlaufend mit allen Beteiligten zu diskutieren. Die APE 2020 ist schon in Planung und wird vom 14. bis 15. Januar wieder in Berlin stattfinden.

Mehr zum Programm und Videoaufzeichnungen auf www.ape2019.eu

Deskriptoren: Tagung, APE 2019, Veröffentlichungswesen, Elektronisches Publizieren, Verlag, Wissenschaftliches Arbeiten, Open Access, Open Science

Elgin Helen Jakisch

U\&B Interim-Services

Berlin

jakisch@ub-interim.de 